\title{
Object-based Selection of Irrelevant Features Is Not Confined to the Attended Object
}

\author{
Carsten N. Boehler ${ }^{1,3}$, Mircea A. Schoenfeld ${ }^{1,2}$, \\ Hans-Jochen Heinze ${ }^{1,2}$, and Jens-Max Hopf ${ }^{1,2}$
}

\begin{abstract}
Attention to one feature of an object can bias the processing of unattended features of that object. Here we demonstrate with ERPs in visual search that this object-based bias for an irrelevant feature also appears in an unattended object when it shares that feature with the target object. Specifically, we show that the ERP response elicited by a distractor object in one visual field is modulated as a function of whether a task-irrelevant color of that distractor is also present in the target object that is presented in the opposite vi-
\end{abstract}

\section{INTRODUCTION}

Objects have been proposed to represent a basic unit of attentional selection. This has been suggested on the basis of numerous behavioral observations (Scholl, 2001), including the seminal "same-object advantage," which refers to the fact that the discrimination of multiple object properties is better when they concern one object versus different objects (Duncan \& Nimmo-Smith, 1996). Influential for conceptualizing the role of attention in linking features with objects has been the notion of integrated competition (Duncan, Humphreys, \& Ward, 1997; Desimone \& Duncan, 1995), which posits that attention selects an object among competing distractors by establishing a topdown competitive bias for properties or actions associated with the relevant object. Competition is suggested to be integrated in the sense that the competitive bias affects all sensory-motor brain systems coding the object and that once an object received competitive advantage with respect to one property, the advantage transfers to other associated properties. Attention is conceived of being focused after the object has transferred its processing bias to all, even task-irrelevant features (Chen \& Cave, 2006). Indeed, neurophysiological studies have provided broad support for this by showing that task-irrelevant object features gain privileged neural processing simply by virtue of being part of the attended object (Katzner, Busse, \& Treue, 2009; Wannig, Rodriguez, \& Freiwald, 2007; Schoenfeld et al., 2003; O’Craven, Downing, \& Kanwisher, 1999; Valdes-

${ }^{1}$ Leibniz Institute for Neurobiology, Magdeburg, Germany, ${ }^{2}$ Ottovon-Guericke University, Magdeburg, Germany, ${ }^{3}$ Duke University, Durham, North Carolina sual field. Importantly, we find this modulation to arise with a delay of approximately $80 \mathrm{msec}$ relative to the N2pc-a component of the ERP response that reflects the focusing of attention onto the target. In a second experiment, we demonstrate that this modulation reflects enhanced neural processing in the unattended object. These observations together facilitate the surprising conclusion that the object-based selection of irrelevant features is spatially global even after attention has selected the target object.

Sosa, Bobes, Rodriguez, \& Pinilla, 1998). Notably, such object-controlled biasing of features is equivalent to the notion that attention serves to bind features for target identification put forward by influential theories on visual search (Wolfe \& Bennet, 1997; Treisman, 1988), which posit that it is focal attention that establishes the unity of an object by linking loosely bundled features (object file), thereby making their presence in the object consciously reportable (Wolfe \& Bennet, 1997; Kahneman, Treisman, \& Gibbs, 1992; Treisman, 1988; Kahneman \& Treisman, 1984).

Here we provide insights into the nature and the relative time course of neural operations underlying object integration in visual search with ERPs, which permit us to assess brain activity reflecting attentional selection in visual cortex with high temporal resolution (Woodman \& Luck, 1999; Luck \& Hillyard, 1994). The reported experiments are specifically designed to assess whether the objectbased selection of task-irrelevant features is an operation confined to the attended object or alternatively a global operation and not confined to the attended object, analogous to previous demonstrations of global selection of task-relevant object features (Zhang \& Luck, 2009; Hopf, Boelmans, Schoenfeld, Luck, \& Heinze, 2004; Saenz, Buracas, \& Boynton, 2002; Treue \& Martinez Trujillo, 1999; Motter, 1994). To this end, the ERP response to a task-irrelevant feature of an unattended object is analyzed as a function of this feature being present or absent in the search target. If the selection of task-irrelevant features is global, we predict ERP effects of this selection to arise in the unattended object. Alternatively, if the object-based selection of irrelevant features remains confined to the attended object, no 
such ERP effects should be seen. For an analysis of the temporal dynamics of the ERP effects, we take reference to the N2pc component - an ERP modulation that is known to reliably index the operation of focusing attention onto the target item in visual search (Hopf et al., 2000; Woodman \& Luck, 1999; Luck, Girelli, McDermott, \& Ford, 1997). Specifically, we take the N2pc to mark the time range of object integration by attention, with the prediction that objectbased effects on feature selection should arise after the onset of the N2pc.

We show in a first experiment that the object-based selection of an irrelevant feature value (of color) establishes, in fact, a global bias for that value in an unattended object (bearing that particular feature value). The bias appears within $80 \mathrm{msec}$ after the onset of the N2pc and therefore the operation of focusing attention onto the target item in visual search. A further experiment demonstrates that this feature bias reflects enhanced neural processing in the unattended object. Our observations together indicate that object-based selection imposes its biasing effect on irrelevant features within tens of milliseconds and that the effect arises as a spatially global operation not confined to the attended object.

\section{METHODS}

\section{Participants}

Sixteen subjects (13 women, mean age $=24.8$ years) took part in Experiment 1, and 13 subjects (12 women, mean age $=25.8$ years) participated in Experiment 2. All subjects were neurologically normal students of the University Magdeburg with normal or corrected-to-normal visual acuity and normal color vision. All participants gave informed consent and were paid for participation. The experiments were approved by the ethics committee of the University Magdeburg.

\section{Stimuli}

\section{Experiment 1}

Each search frame consisted of two three-dimensional spheres $\left(2.5^{\circ}\right.$ diameter $)$, one presented in the left and one in the right visual field (VF; centered $\pm 4.5^{\circ}$ lateral and $2^{\circ}$ below fixation) for $700 \mathrm{msec}$. The left and the right halves of each sphere were drawn in a different color taken from an array of four colors (red, green, blue, and yellow; Figure $1 \mathrm{~A}$ and $\mathrm{B}$ ). At the start of each trial block, a target color (red in Figure 1) was designated, which appeared on each trial in one of the half-spheres either in the left or the right VF. The other colors were assigned to the remaining half-spheres in a random fashion, but with the requirement that on $50 \%$ of the trials, one of the nontarget colors appeared in both VFs (green in Figure 1A). On the remaining trials, all four half-spheres were assigned a differ-

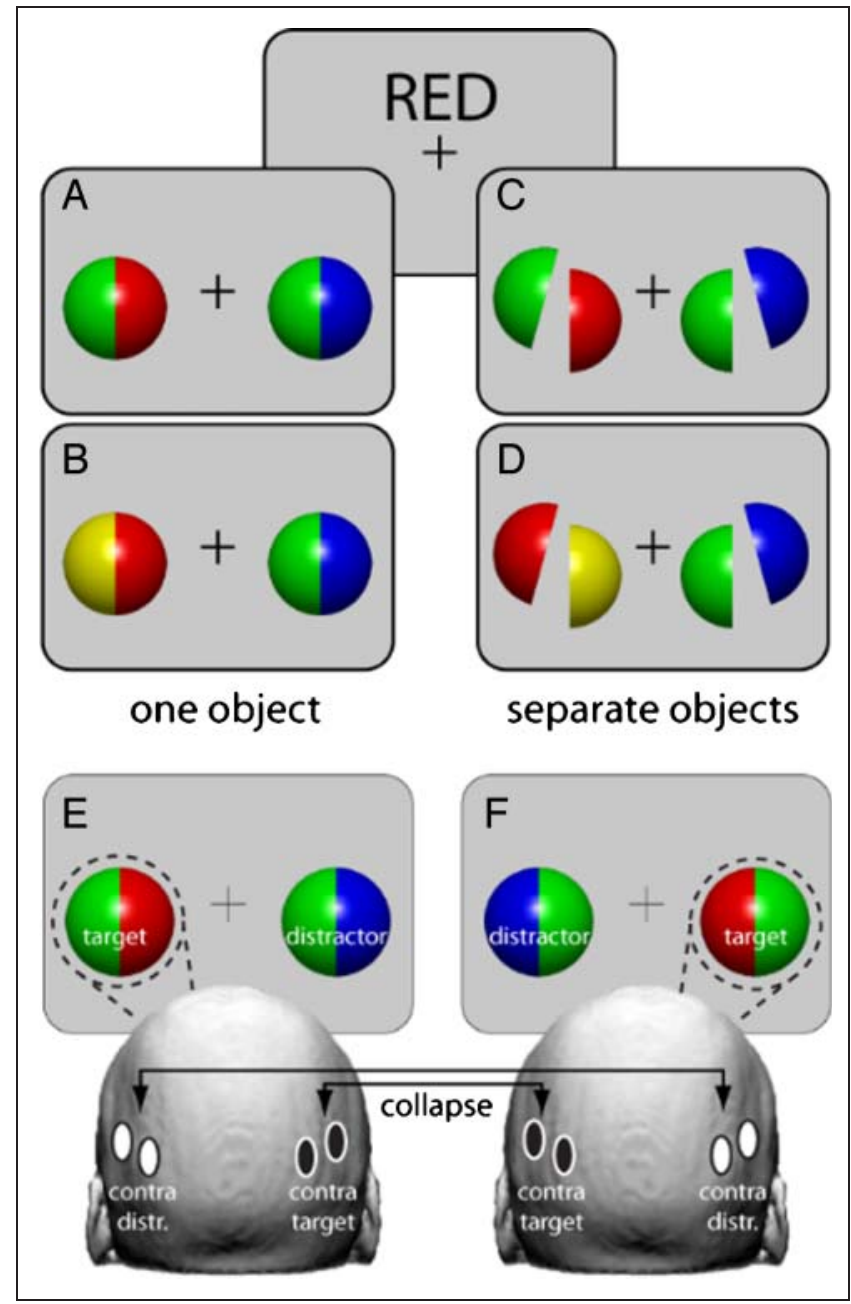

Figure 1. Stimulus setup of Experiment 1. (A and B) Example search arrays of the one object condition. (A) An array where the irrelevant color (green) is contained in both the target (red/green) and the distractor object (blue/green). (B) An array that shows a situation where the irrelevant color (green) is not contained in the target (red/yellow). (C and D) Example search arrays of the separate object condition. Spheres were cut apart and slightly displaced and rotated. The spatial distribution of color is identical to the one object condition in panels A and B, except that colors now appear on separate objects. (E and F) Data assessment in Experiment 1. Because of the contralateral retinotopical organization of the visual system, the lateralized presentation of an item is associated with ERP activity over the contralateral occipital hemisphere. The ERP responses reflecting the distractor and the target are therefore separable in the present experiment as they correspond with opposite-hemisphere electrodes (white and black circles). Activity from electrodes reflecting the distractor (white) and electrodes reflecting the target (black) was collapsed across trials with the target presented in the left or the right VF (see arrows).

ent color (Figure 1B). Target color was changed across trial blocks such that each color served equally often as target. A comparison of the ERP response with the spheres under those conditions permitted us to assess the effect of an irrelevant color in the nontarget sphere as a function of whether that color is contained in the attended sphere or not. Figure $1 \mathrm{~A}$ and $\mathrm{B}$ illustrates those conditions with red as 
target color. The task-irrelevant green in the nontarget object presented in the right VF the (green/blue sphere) is either present in the target object in the left VF (Figure 1A) or not (Figure 1B). To assess the effect of object-based selection, in a different type of trial blocks, the half-spheres were cut apart and slightly rotated relative to each other (one half-sphere moved upward by $0.4^{\circ}$ and rotated outward by $15^{\circ}$ of directional) such that each color belonged to a separate object (separate objects condition; Figure 1C and D). The spatial distribution of colors across the VFs was identical to the trial blocks with intact spheres (one object condition).

\section{Experiment 2}

The stimuli were identical to the one object condition of Experiment 1 , except that on $50 \%$ of the trials, one irrelevant color of the nontarget sphere was replaced by a checkerboard luminance modulation of that color (the probe) 300 msec after stimulus onset (frame-probe trials) as illustrated in Figure 4A. On the remaining trials, no probe was presented (frame-only trials). The inclusion of frame-only trials permitted to assess the brain response to the probe proper (referred to as probe response) by subtracting the response to frame-only trials from that of corresponding frame-probe trials. The check size of the probe was $0.4^{\circ}$, with the luminance value of the bright and dark checks set to the highest and lowest luminance value on the corresponding three-dimensional half-sphere, respectively.

\section{Procedure}

\section{Experiment 1}

Subjects' tasks were to fixate the center of the screen and to discriminate the direction of convexity (two-alternative button press, index/middle finger of the right hand) of the half-sphere drawn in the target color. The spheres were presented for $700 \mathrm{msec}$ in a trial sequence with SOAs randomized between 1700 and 2200 msec. Subjects performed a total of eight trial blocks with each of the four colors serving as the target color two times in the whole experiment, once in a trial block of the one object and once of the separate objects condition. One trial block consisted of 192 trials, which amounted to a total of 384 trials with the same irrelevant color appearing in both VF and 384 trials where all irrelevant colors were different both for the one object and the separate object condition.

\section{Experiment 2}

Subjects were instructed that the probe stimuli were completely task irrelevant and were to be ignored. The subjects' task and all other experimental parameters were identical to the one object condition of Experiment 1. Subjects performed a total of eight trial blocks, with each block containing 96 frame-only and 96 frame-probe trials, half of them probing an irrelevant color in the nontarget object that appeared in both VFs and half of them probing an irrelevant color only present in the nontarget VF.

\section{ERP Recording and Analysis}

\section{Experiment 1}

The EEG was obtained from 32 electrode positions of the standard 10-20 montage system and its 10-10 extension (American Electroencephalographic Society, 1994). Recordings were performed using a 32-channel Synamps amplifier system (NeuroScan Inc., Herndon, VA). The signal was referenced against the right mastoid, low-pass filtered at DC-50 Hz, and then digitally sampled at a rate of $254 \mathrm{~Hz}$. Horizontal eye movements were recorded by a bipolar EOG montage with two electrodes behind the lateral orbital angles, vertical eye movements with an electrode below the right orbital limb. Eye blinks and movement artifacts were removed by discarding trials exceeding a threshold of $100 \mu \mathrm{V}$. Data were then algebraically rereferenced against a weighted mean of the left and right mastoid and submitted to subsequent signal averaging.

For each subject, separate ERP averages were computed for trials with the same irrelevant color appearing in both VF and trials with irrelevant colors differing between VFs, with the responses collapsed across the four different colors. Averaging was first performed separately for nontarget spheres presented in the left and right VF. Note that because lateralized objects elicit their response mainly in the hemisphere contralateral to the half-field of presentation, activity reflecting the distractor sphere could be separated from activity reflecting the target sphere. Figure $1 \mathrm{E}$ and $\mathrm{F}$ illustrates this logic. In Figure 1E, the distractor sphere appears in the right VF, with a corresponding response in the left-hemisphere electrode (white), whereas the response specific to the target is measured at the opposite righthemisphere electrode (black). The mirror-image situation is shown in Figure $1 \mathrm{~F}$, where the target sphere appears in the right VF. For data analysis, the ERP responses reflecting the distractor and the target were collapsed across left and right VF targets such that waveforms recorded from right-hemisphere electrodes (P8, PO8) contralateral to a nontarget in the left VF were combined with waveforms recorded from left-hemisphere electrodes (P7, PO7) contralateral to a nontarget in the right VF (see arrows in Figure 1E and F). Waveforms recorded from electrodes contralateral to a target were combined analogously, resulting in ERP averages corresponding to principal electrode sites ( $\mathrm{P} 7 / 8$, $\mathrm{PO} 7 / 8$ ) contralateral to the distractor sphere (contra distr.) or the target sphere (contra target). For statistical validation of the data, repeated measures ANOVA (rANOVA) were performed on mean voltage amplitudes in selected time ranges. The time course of ERP differences was determined on the basis of sliding $t$ tests (a 20-msec window sliding time sample by time sample), with the criterion that the 
first significant difference $(p<.05)$ in a row of at least five consecutive significant samples marks the temporal onset of the ERP difference.

\section{Experiment 2}

Principal data analysis was identical to Experiment 1 . The ERP response to the probe was obtained by subtracting waveforms elicited by frame-only trials from waveforms elicited by frame-probe trials. Those difference waveforms (probe response) were separately computed for trials with the same irrelevant color appearing in both VF and trials with irrelevant colors differing between VFs. The probe response for probes in the left and right VF were collapsed according to the logic illustrated in Figure 1E and F. Again, responses were collapsed across the different colors.

\section{RESULTS}

\section{Experiment 1}

\section{Behavioral Performance}

Figure 2 summarizes RT and response accuracy of the one object and separate objects condition separately for trials with the same nontarget color presented in both VFs (black) and for trials with nontarget color differing between VFs (gray). Performance accuracy was generally high and RTs relatively fast under all conditions, but there was no visible difference between experimental conditions. A two-way rANOVA with the factors number of objects (one/separate) and nontarget color distribution (nontarget color the same in both VFs/nontarget color different between VFs) confirmed this impression. There was neither a main effect of number of objects, accuracy, $F(1$, $15)=0.31, p=.58$, RT, $F(1,15)=0.18, p=.68$, nor a main effect of nontarget color distribution, accuracy, $F(1$, $15)=0.001, p=.98$, RT, $F(1,15)=0.62, p=.44$. There was also no significant interaction of number of Objects $\times$ Nontarget color distribution, accuracy, $F(1,15)=0.53$, $p=.48$, RT, $F(1,15)=2.6, p=.14$. In sum, the presence versus absence of an irrelevant target feature in the unattended object did not influence performance measures. In the one object condition, there was a minimal RT increase ( $4 \mathrm{msec}$ ) of the former versus the latter that was not present in the separate objects condition, but the respective interaction was not significant.

\section{ERP Results}

Figure 3A shows average ERP waveforms to trials with a nontarget color presented in both VFs (dashed traces) overlaid on waveforms to trials where nontarget colors differed between VFs. For the one object condition (upper row), a clear and statistically significant amplitude difference is visible between 270 and 500 msec after stimulus onset at the electrode site contralateral to the unattended sphere (red area between traces), mean amplitude difference $F(1$, $15)=6.82, p<.02$. No amplitude difference is observed for electrode sites contralateral to the attended object, $F(1$, $15)=0.24$. The topographical map of this difference shown on the right in Figure 3A shows a maximum over the lateral occipital cortex contralateral to the distractor object. Importantly, the analogue comparison of waveforms for the separate object condition (lower row) does not show such modulation contralateral to the unattended object. The ERP response to the split spheres showed generally larger P1/N1 responses (presumably reflecting the higher frequency content of the stimuli due to the sharp edges), but there was no amplitude difference at all with respect to whether any color in the nontarget VF matched the irrelevant color in the target VF. This rules out the possibility that differences in the spatial distribution of color per se were responsible for the ERP difference. Both experimental conditions taken together clearly suggest that the observed ERP modulation for irrelevant features in unattended objects is
Figure 2. Behavioral results of Experiment 1. Average performance accuracy (\% correct responses, left) and RT (msec, right). Data are separately averaged for the one and separate object condition as well as for trials with the target color presented in both VFs (black) versus in the target VF only (gray).

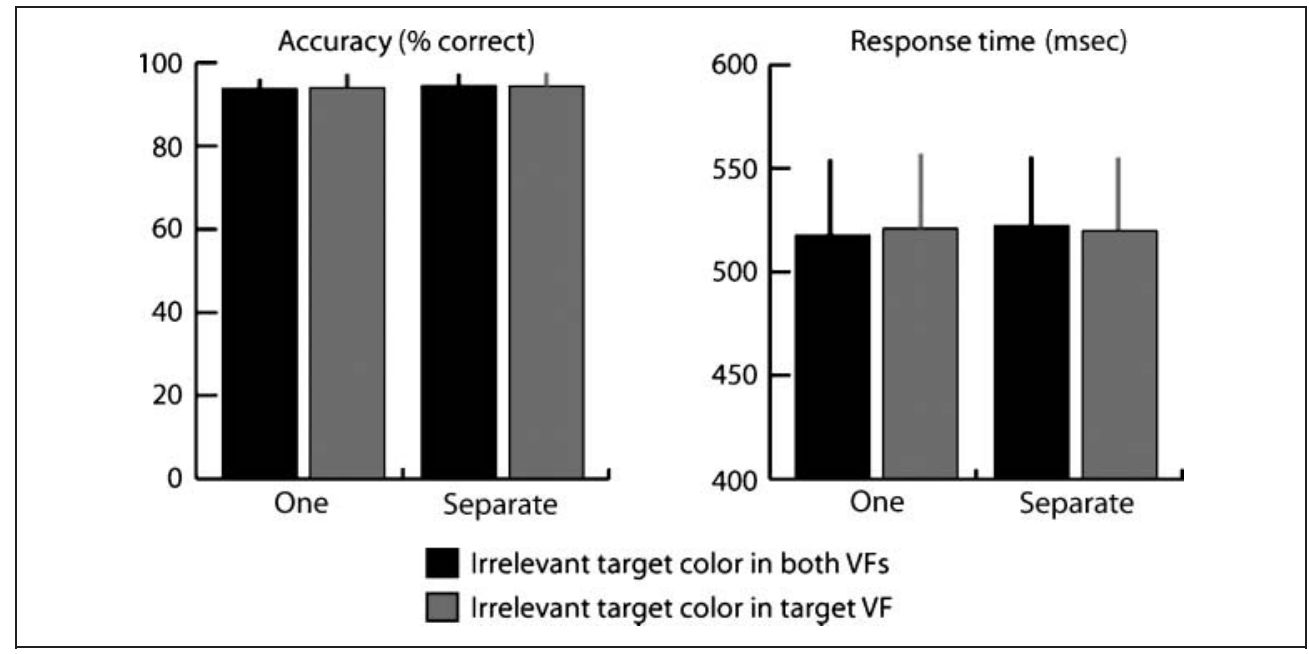


Figure 3. ERP results of Experiment 1. (A) ERP waveforms elicited by the one and separate objects condition at occipital hemisphere electrodes (P7, PO7/P8, PO8) ipsilateral (ipsi) or contralateral (contra) to the unattended object(s). Waveforms were collapsed across left and right VF targets. The scalp distribution on the right shows the IFE (waveform-difference irrelevant target color in both VFs minus irrelevant target color in target VF only) at $400 \mathrm{msec}$ after search frame onset. (B) Waveforms and scalp distribution of the N2pc response (blue area between traces) of the one object condition. The top map shows the N2pc (left VF minus right VF target difference) at $250 \mathrm{msec}$ after search frame onset. The blue horizontal bar highlights the time range in which the N2pc was significant, and the red horizontal bar highlights the time course of the IFE seen in the one-object condition.

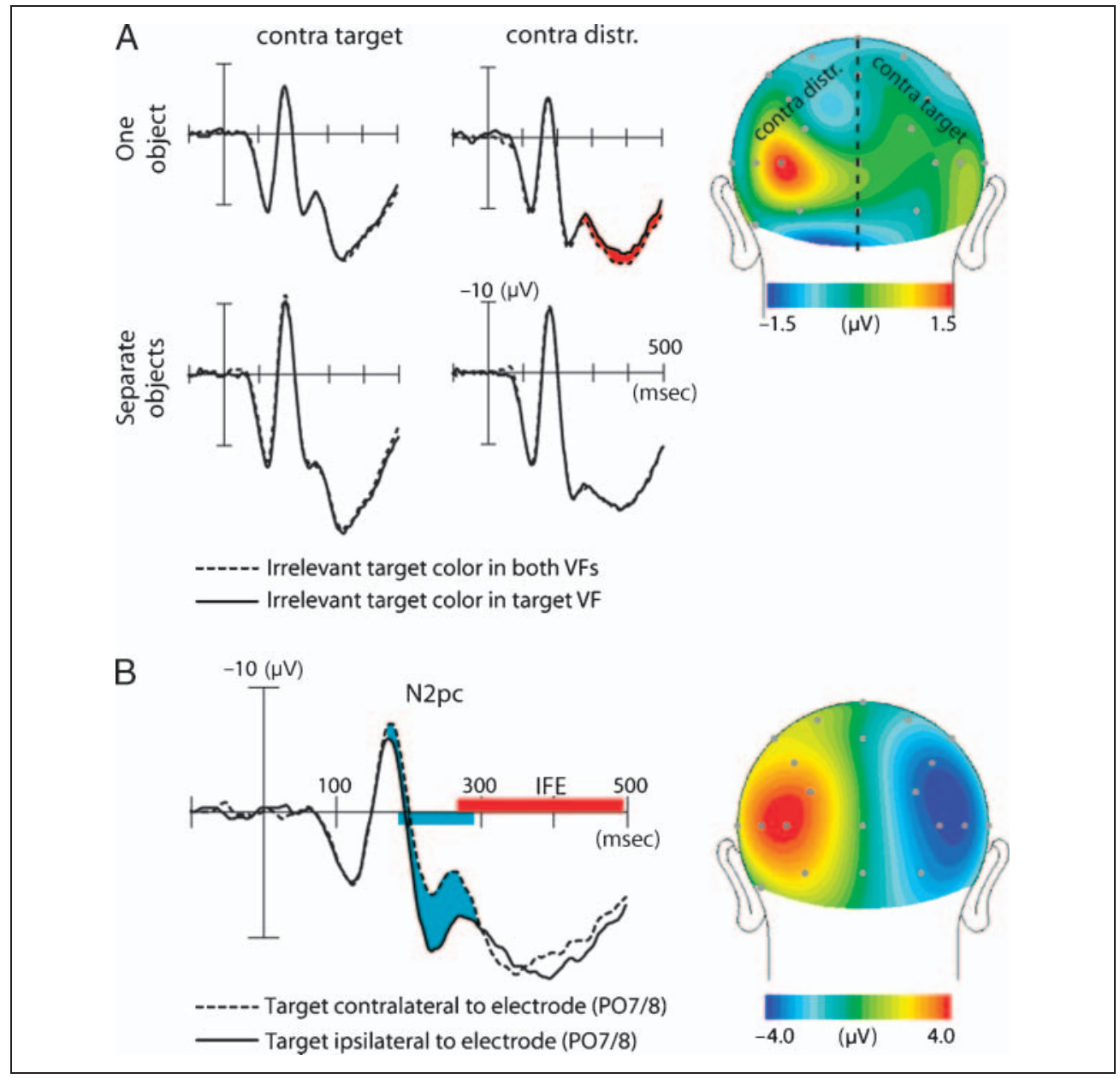

mediated by an object-based mechanism. In the following, we will refer to this modulation as irrelevant feature effect (IFE).

An important point to clarify with ERPs is how the time course of the IFE compares with the time course of focusing spatial attention onto the target object. An onset before the operation of focusing attention would suggest that the IFE reflects a spatially global effect that arises at a stage when attention has not yet accomplished the integration of features at the target location. Such observation would be akin to our previous demonstration of a global locationindependent selection of relevant features before object selection in visual search (Hopf et al., 2004). In contrast, a delayed onset of the IFE relative to spatial focusing would suggest that irrelevant features are "noticed" at unattended locations after attention has integrated or bound features at the target location, consistent with object-based selection. To address this issue, we analyzed the N2pc component (a negative deflection in the N2 time range over the posterior cortex contralateral to the target item) that is known to reflect the operation and time course of focusing attention onto the target object in visual search (Hopf et al., 2000; Woodman \& Luck, 1999; Luck et al., 1997). The N2pc is typically derived by comparing the ERP response with search frames with the target presented in the contralateral versus the ipsilateral VF relative to a lateralized posterior electrode (e.g., PO7/PO8). Figure 3B shows waveforms elicited by contralateral (dashed) and ipsilateral target spheres (solid) of the one object condition (no IFE trials only), with the N2pc effect highlighted as blue area between traces (data collapsed over left and right VF targets). The map on the right shows the topographical distribution of the N2pc effect (target-left minus target-right VF difference), which displays the typical pattern, that is, opposite polarity maxima over the left and right occipito-parietal scalp (Hopf et al., 2000; Girelli \& Luck, 1997). In the waveforms, the time course of the IFE as validated by sliding $t$ tests ( $p<.05$, see Methods) is indicated as red horizontal bar. Apparently, the N2pc arises significantly earlier than the onset of the IFE. Sliding $t$ tests $(p<.05)$ revealed that the N2pc was significant between 190 and 290 msec after stimulus onset (blue horizontal bar), whereas the IFE was not significant before $270 \mathrm{msec}$ (a sliding $t$ test analysis of the IFE on waveforms contralateral to the target revealed no significant effect). Thus, the IFE appears with a delay of approximately $80 \mathrm{msec}$ relative to the onset of the N2pc, that is, the operation of focusing spatial attention onto the target.

In sum, Experiment 1 shows that the object-based bias for irrelevant features contained in the target object appears 
in unattended objects after attention has been focused onto the target object. The global nature of this bias is notable in view of notions of the feature integration theory (FIT) (Treisman \& Gormican, 1988; Treisman \& Gelade, 1980). According to FIT, one would expect, at least at first glance, that because attention serves to integrate features of the attended object, unattended objects should not display delayed effects of global feature facilitation. However, it is possible that the object-based facilitation of the irrelevant color is confined to the target object, but because this bias increases competition with the target color, the distractor color is subjected to neural attenuation, which may then operate in a global way. In fact, in a later development of the FIT, it was proposed that visual search involves an operation on the basis of a global and parallel reduction of the relative prominence of distractor locations bearing a common feature (Treisman \& Sato, 1990). In this scenario, the IFE could represent some form of N2pc response reflecting distractor attenuation because of the increased need to suppress interference from the irrelevant target color in the unattended object. In fact, a recent ERP study revealed that the N2pc may be composed of separate lateralized subcomponents with a negativity contralateral to the search target reflecting target processing and a relative positivity contralateral to the distractor indexing distractor suppression (distractor positivity; Hickey, Di Lollo, \& McDonald, 2009). The relative polarity of the IFE would indeed be compatible with such distractor positivity. Therefore, it is crucial to clarify whether the IFE reflects facilitated or attenuated neural activity in the unattended object. This question was addressed in Experiment 2 by employing passive probe stimulation.

\section{Experiment 2}

Experiment 2 was identical to the first experiment (one object condition), except that on $50 \%$ of the trials, one half of the nontarget sphere was replaced by a short $(50 \mathrm{msec})$ checkerboard luminance modulation of its color $300 \mathrm{msec}$ after stimulus onset. This checkerboard modulation was completely task irrelevant and served as a probe into the passive responsivity of the cortex representing the distractor location. The size of the ERP response elicited by this probe was analyzed as a function of whether the probed color was or was not contained as irrelevant color in the target object (Figure 4A).

Figure $4 \mathrm{~B}$ illustrates the time course and topographical distribution of the IFE and N2pc from no-probe trials in Experiment 2. The amplitude of the modulation representing the IFE is somewhat smaller than in Experiment 1 but shows a significant effect between 320 and $420 \mathrm{msec}, F(1$, $12)=5.22, p<.05$. Statistical testing of waveform differences contralateral to the target revealed no significant effect. Again, the IFE effect is delayed relative to the time course of the N2pc. Sliding $t$ tests (see Methods) revealed that the N2pc was significant $(p<.05)$ between 210

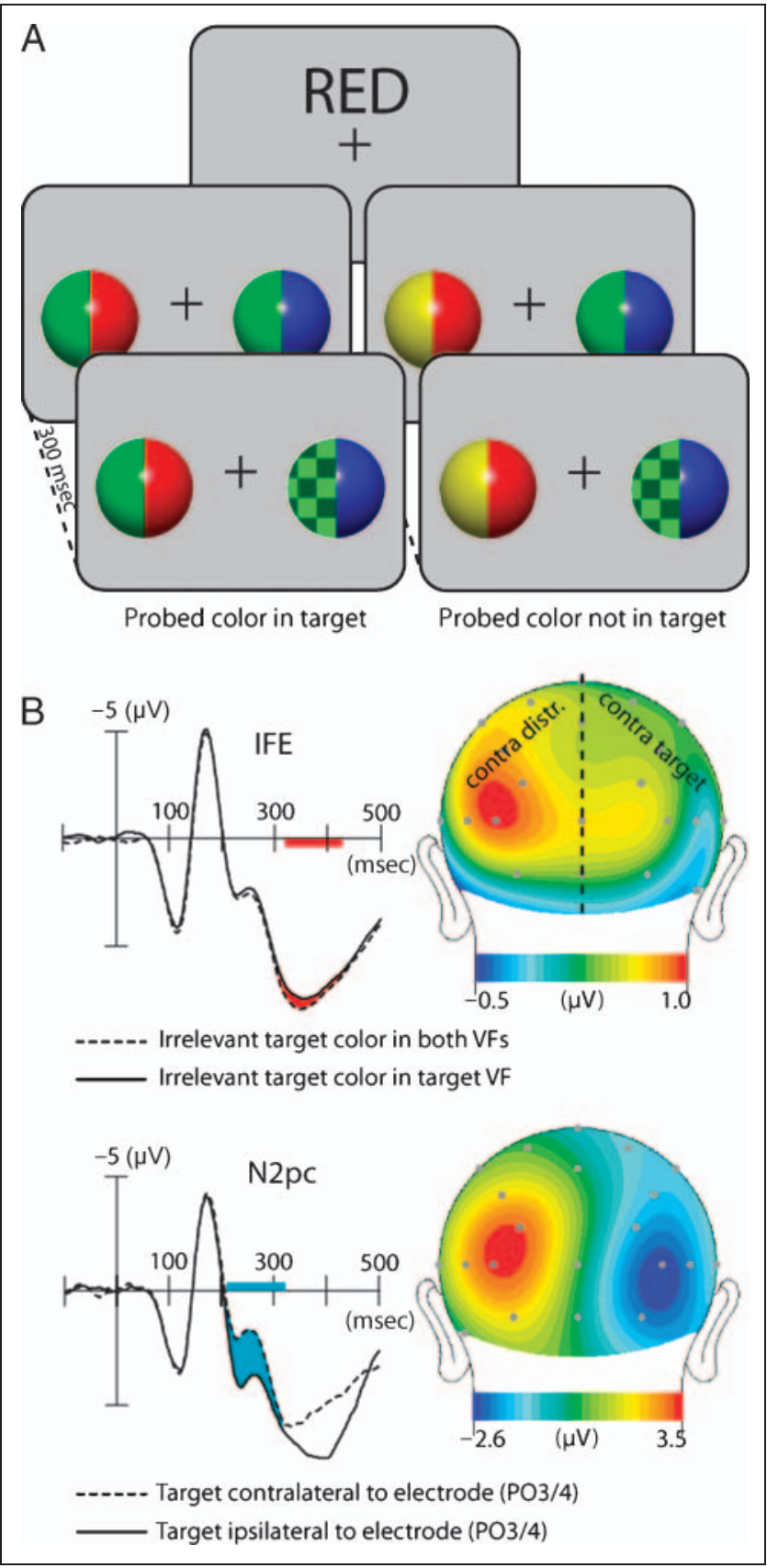

Figure 4. Stimulus setup and results of Experiment 2. (A) Search frames were identical to the one object condition except that a probe (a checkerboard modulation of color) replaced one half of the unattended object 300 msec after frame onset on $50 \%$ of the trials. The probed color (green) could either be part of the attended object (the sphere containing red; left) or not (right)). (B) Waveforms and voltage distributions showing the IFE effect (upper row) and the N2pc response (lower row) of Experiment 2.

and $320 \mathrm{msec}$, whereas the IFE was not significant before $320 \mathrm{msec}$.

Figure 5 shows the probe response for the probed nontarget color being contained (red trace) versus being not contained (black trace) in the target sphere. Apparently, the former is enhanced relative to the latter. A statistical comparison of the mean amplitude (160-350 msec) of 
the probe response (probe minus no-probe difference) between conditions yielded a significant difference, $F(1$, $12)=5.6, p<.05$. This enhancement is further illustrated in the topographical maps, which show the distribution of the probe minus no-probe difference collapsed over left and right VF probes at $270 \mathrm{msec}$. In the maps, the left hemisphere is arbitrarily chosen to show responses contralateral to the probe, whereas the right hemisphere shows responses contralateral to the target. Obviously, the topographical maximum of the probe response appears over the left occipital cortex, which is contralateral to (and therefore retinotopically consistent with) the probe's location. It should be noted that the enhancement of the probe response arises as increased negativity of early components $(\mathrm{N} 1, \mathrm{~N} 2)$ reflecting the sensory processing of the probe. In a later time range $(>300 \mathrm{msec})$ corresponding with the $\mathrm{P} 300$, the probe response is also more negative but would actually indicate a reduced $\mathrm{P} 300$ response. It is possible that this reduction appears because of a prolonged negative enhancement that overlaps with the $\mathrm{P} 300$ time range, thereby producing a relative reduction of the latter component. Alternatively, it is possible that the probe stirred "extra distraction" when probing the distractor color contained in the target, which may then have led to an extra N2pc response to counter this distraction. Aside from those possibilities, the important observation is that the IFE is associated with an increased sensory ERP response to the probe, sug-

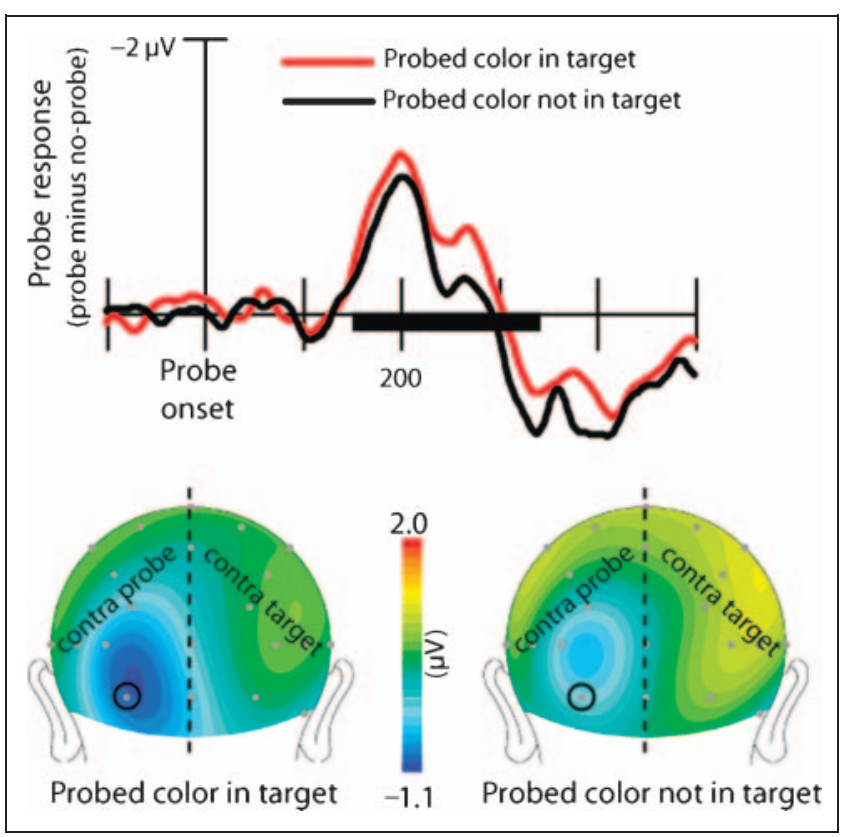

Figure 5. Probe-response data of Experiment 2. ERP response elicited by the probe as a function of the probed color being contained in the target (red trace) or not (black trace). Shown are difference waves (probe minus no-probe trials). The waveforms were taken from the electrode site highlighted in the maps below (black circle). The topographical maps show the distribution of the probe response at 270 msec after probe onset when the probed color was part of the target (left) or was not contained in the target (right). gesting that the IFE reflects enhanced sensory processing of the irrelevant feature in the unattended object.

\section{DISCUSSION}

The present data show that attending to a particular color of the target object facilitates the processing of another task-irrelevant color simply by virtue of the fact that this color is contained in the attended object. Such processing bias $^{1}$ for irrelevant object properties has been taken to witness the operation of object-based attention (Katzner et al., 2009; Wannig et al., 2007; Sohn, Papathomas, Blaser, \& Vidnyanszky, 2004; Schoenfeld et al., 2003; O'Craven et al., 1999; Valdes-Sosa et al., 1998). The present data add new evidence in revealing the time course of this biasing effect relative to the time course of focusing spatial attention onto the target. The IFE appeared with a delay of 80 msec relative to the onset of the N2pc-a component that is known to index the time course of attentional focusing onto the target object in visual search (Woodman \& Luck, 1999; Luck \& Hillyard, 1994). A delay relative to the N2pc is consistent with the object-based mediation of the IFE, as one would expect that it takes time for attention to propagate the modulatory bias from the relevant to the irrelevant feature. In fact, a delay of this order was also seen in a recent investigation of object-based integration of color and motion (Schoenfeld et al., 2003). In this study, attention to color of an object was found to facilitate irrelevant motion of that object with a delay of 40-60 msec.

The more important observation reported here is that the object-based bias of irrelevant feature processing is not confined to the attended object but appears in the distractor object, suggesting that irrelevant features of an attended object become globally linked in a visual scene (IFE). As revealed by Experiment 2, the IFE represents enhanced processing of the irrelevant feature in the distractor. Some evidence consistent with such linking has been recently provided for color and motion (Melcher, Papathomas, \& Vidnyanszky, 2005; Sohn, Chong, Papathomas, \& Vidnyanszky, 2005). Melcher et al. (2005) observed that a motion prime presented outside the spatial focus of attention facilitated subsequent motion coherence sensitivity when the prime's color matched the color of attended dot motion in the focus of attention. This observation, referred to as implicit attentional selection, demonstrated that attention to color transferred its sensory facilitation to the irrelevant motion feature at unattended item locations. Likewise, Katzner et al. (2009) report attention effects on monkey MT cells consistent with such global object-based selection irrelevant features. Firing enhancements of MT cells were observed when the color (not the motion) of dots moving in the cell's preferred direction was attended outside its receptive field. It should be noted that implicit attentional selection shown by Melcher et al. and Katzner et al. refers to a linking across different feature dimensions (color and motion), whereas the present study demonstrates such linking for the first time between different values of one 
feature dimension (color). Finally, Chen and Cave (2006) report behavioral effects on response compatibility suggesting object-based selection of irrelevant features in distractor items. In their experiments, subjects were cued to discriminate a color or an orientation pop-out item, both simultaneously presented in a search array. The response alternatives for the color and orientation discrimination overlapped, and performance was analyzed as a function of whether the response associated with the irrelevant distractor (on a different trial) was compatible or incompatible with the response required to discriminate the target. Effects of response compatibility were observed, indicating that the irrelevant feature of the distractor was processed to the extent it interfered with the response tendency to the target. Note that the observations of Chen and Cave provide clear evidence for global selection of task-irrelevant object features but that they differ from the present data as they demonstrate the global linkage of selection via response mapping. In the present study, response compatibility effects were not involved, and the IFE was also not mirrored on the behavioral level.

The present observation that object-based attention biases irrelevant features in unattended objects after spatial attention has selected the target object has implications for conceptualizing the role of feature and spatial attention in visual search. A key notion of influential theories (Cave, 1999; Treisman, 1998; Kahneman et al., 1992; Wolfe, Cave, \& Franzel, 1989; Treisman \& Gelade, 1980) regarding the role of focal attention is that which mediates the correct linking/binding of features (relevant or irrelevant ones) to the object file and thereby instantiates the (reportable) identity and coherence of an object. Focal attention is assumed to make explicit that features are features of a particular object and not of other objects (for a similar notion in the framework of the FINST model, see Pylyshyn, 2001). In view of this interpretation, the present observations are notable as they suggest that associated attentional operations in the feature domain are nevertheless global and not confined to an attended object. This somewhat unexpected finding is in contrast to the observation that the selection of task-relevant features in visual search is global and appears before (30 msec) spatial focusing as reflected by the N2pc (Hopf et al., 2004). Global selection of taskrelevant features is predicted, as it is plausible that those features are decoded before the identification of the target, with this decoding operating in a global way to guide the spatial allocation of attention to potential target locations.

What then is the significance of the global object-mediated facilitation of irrelevant features reported here? At a first glance, this effect appears to be counterproductive in terms of building a coherent representation of an attended object. It may, however, be useful in other important respects. For example, coding object-coherence across occlusions (Behrmann, Zemel, \& Mozer, 1998) may benefit from globally linking unattended features of the target object. Because of object discontinuity, the initial preattentional segmentation of an object may be incomplete, and a global bias of its unattended features elsewhere could then provide further cues for revising the incorrect segmentation. Moreover, this mechanisms may facilitate the grouping of distributed objects as for example in multi-item tracking (Yantis, 1992). Finally, such global bias may underlie the (parallel) marking of distractor locations for suppression in visual search, in particular of those locations containing irrelevant features that interfere with target discrimination. In fact, such feature-based grouping for inhibition-called feature segregation and inhibition strategy - has been proposed by Treisman and Sato (1990) to extend the feature integration model. According to this extension, attention in addition to a spatially serial mode may also operate by globally controlling the relative activation of master-map locations via linkage to feature maps. It is suggested that distractor locations containing a distinct irrelevant feature can be attenuated by parallel inhibition from a corresponding feature map, which facilitates search because of a reduction of search space. Experiment 2 revealed that the global bias for the irrelevant feature represents neural enhancement, which seems not directly compatible with that notion.

\section{Conclusion}

The present data provide evidence in support of a seminal prediction of integrated competition (Duncan et al., 1997), namely, that an attended object controls the biasing of its irrelevant features to gain competitive advantage in a visual scene. However, we show that this bias is established in a spatially global manner within approximately $80 \mathrm{msec}$ after attention started to integrate the attended object. Our findings extend and complement considerable neurophysiological evidence, indicating that explicitly attended features are globally selected (Zhang \& Luck, 2009; Hopf et al., 2004; Saenz et al., 2002; Treue \& Martinez Trujillo, 1999; Motter, 1994). Those and the present observations together may be taken to foster an intriguing hypothesis: Biasing feature selection (top-down or implicit via object integration) is inherently global in the visual system, even if relevant location information is available to constrain selection and focal attention has built an integrated object representation.

\section{Acknowledgments}

This research was made possible by grant no. SFB779/TPA1.

Reprint requests should be sent to Jens-Max Hopf, Department of Neurology, Otto-von-Guericke University of Magdeburg, and Leibniz Institute for Neurobiology, Leipziger Strasse 44, 39120 Magdeburg, Germany, or via e-mail: jens-max.hopf@medizin.unimagdeburg.de.

\section{Note}

1. It should be noted that it is conceivable that the feature bias indexed by the IFE reflects the global relative attenuation of the irrelevant color present in the unattended but not the attended object. Although this possibility cannot be ruled out 
completely in the present experiments, it is an unlikely interpretation. Explaining our findings in terms of such form of global inhibition would require the rather more complex scenario of object-based selection being selective and explicit regarding irrelevant feature-values not contained in the attended object, which only then could be the basis of selectively subjecting those features to global attenuation.

\section{REFERENCES}

American Electroencephalographic Society. (1994). Guideline thirteen: Guidelines for standard electrode position nomenclature. Journal of Clinical Neurophysiology, 11, 111-113.

Behrmann, M., Zemel, R. S., \& Mozer, M. C. (1998). Object-based attention and occlusion: Evidence from normal participants and a computational model. Journal of Experimental Psychology: Human Perception and Performance, 24, 1011-1036.

Cave, K. R. (1999). The FeatureGate model of visual selection. Psychological Research, 62, 182-194.

Chen, Z., \& Cave, K. R. (2006). When does visual attention select all features of a distractor? Journal of Experimental Psychology: Human Perception and Performance, 32, 1452-1464.

Desimone, R., \& Duncan, J. (1995). Neural mechanisms of selective visual attention. Annual Review of Neuroscience, 18, 193-222.

Duncan, J., Humphreys, G. W., \& Ward, R. M. (1997). Competitive brain activity in visual attention. Current Opinion in Neurobiology, 7, 255-261.

Duncan, J., \& Nimmo-Smith, I. (1996). Objects and attributes in divided attention: Surface and boundary systems. Perception \& Psychophysics, 58, 1076-1084.

Girelli, M., \& Luck, S. J. (1997). Are the same attentional mechanisms used to detect visual search targets defined by color, orientation, and motion? Journal of Cognitive Neuroscience, 9, 238-253.

Hickey, C., Di Lollo, V., \& McDonald, J. J. (2009). Electrophysiological indices of target and distractor processing in visual search. Journal of Cognitive Neuroscience, 21, 760-775.

Hopf, J. M., Boelmans, K., Schoenfeld, A., Luck, S. J., \& Heinze, H.-J. (2004). Attention to features precedes attention to locations in visual search: Evidence from electromagnetic brain responses in humans. Journal of Neuroscience, 24, 1822-1832.

Hopf, J.-M., Luck, S. J., Girelli, M., Hagner, T., Mangun, G. R., Scheich, H., et al. (2000). Neural sources of focused attention in visual search. Cerebral Cortex, 10, 1233-1241.

Kahneman, D., \& Treisman, A. (1984). Changing views of attention and automaticity. In R. Parasuraman \& D. R. Davis (Eds.), Varieties of attention (pp. 29-61). New York: Academic Press.

Kahneman, D., Treisman, A., \& Gibbs, B. J. (1992). The reviewing of object files: Object-specific integration of information. Cognitive Psychology, 24, 175-219.

Katzner, S., Busse, L., \& Treue, S. (2009). Attention to the color of a moving stimulus modulates motion-signal processing in macaque area MT: Evidence for a unified attentional system. Frontiers in Systems Neuroscience, 3, 1-8.

Luck, S. J., Girelli, M., McDermott, M. T., \& Ford, M. A. (1997). Bridging the gap between monkey neurophysiology and human perception: An ambiguity resolution theory of visual selective attention. Cognitive Psychology, 33, 64-87.

Luck, S. J., \& Hillyard, S. A. (1994). Electrophysiological correlates of feature analysis during visual search. Psychophysiology, 31, 291-308.
Melcher, D., Papathomas, T. V., \& Vidnyanszky, Z. (2005). Implicit attentional selection of bound visual features. Neuron, 46, 723-729.

Motter, B. C. (1994). Neural correlates of attentive selection for color or luminance in extrastriate area V4. Journal of Neuroscience, 14, 2178-2189.

O’Craven, K. M., Downing, P. E., \& Kanwisher, N. (1999). fMRI evidence for objects as the units of attentional selection. Nature, 401, 584-587.

Pylyshyn, Z. (2001). Visual indexes, preconceptual objects, and situated vision. Cognition, 80, 127-158.

Saenz, M., Buracas, G. T., \& Boynton, G. M. (2002). Global effects of feature-based attention in human visual cortex. Nature Neuroscience, 5, 631-632.

Schoenfeld, M. A., Tempelmann, C., Martinez, A., Hopf, J. M., Sattler, C., Heinze, H. J., et al. (2003). Dynamics of feature binding during object-selective attention. Proceedings of the National Academy of Sciences, U.S.A., 100, 11806-11811.

Scholl, B. J. (2001). Objects and attention: The state of the art. Cognition, 80, 1-46.

Sohn, W., Chong, S. C., Papathomas, T. V., \& Vidnyanszky, Z. (2005). Cross-feature spread of global attentional modulation in human area MT+. NeuroReport, 16, 1389-1393.

Sohn, W., Papathomas, T. V., Blaser, E., \& Vidnyanszky, Z. (2004). Object-based cross-feature attentional modulation from color to motion. Vision Research, 44, 1437-1443.

Treisman, A. (1988). Features and objects: The Fourteenth Bartlett Memorial Lecture. Quarterly Journal of Experimental Psychology, 40A, 201-237.

Treisman, A. (1998). Feature binding, attention and object perception. Philosophical Transactions of the Royal Society of London, Series B, Biological Sciences, 353, 1295-1306.

Treisman, A., \& Gelade, G. (1980). A feature-integration theory of attention. Cognitive Psychology, 12, 97-136.

Treisman, A., \& Gormican, S. (1988). Feature analysis in early vision: Evidence from search asymmetries. Psychological Review, 95, 15-48.

Treisman, A., \& Sato, S. (1990). Conjunction search revisited. Journal of Experimental Psychology: Human Perception and Performance, 16, 459-478.

Treue, S., \& Martinez Trujillo, J. C. (1999). Feature-based attention influences motion processing gain in macaque visual cortex. Nature, 399, 575-579.

Valdes-Sosa, M., Bobes, M. A., Rodriguez, V., \& Pinilla, T. (1998). Switching attention without shifting the spotlight object-based attentional modulation of brain potentials. Journal of Cognitive Neuroscience, 10, 137-151.

Wannig, A., Rodriguez, V., \& Freiwald, W. A. (2007). Attention to surfaces modulates motion processing in extrastriate area MT. Neuron, 54, 639-651.

Wolfe, J. M., \& Bennet, S. C. (1997). Preattentive object files: Shapeless bundles of basic features. Vision Research, 37, 25-43.

Wolfe, J. M., Cave, K. R., \& Franzel, S. L. (1989). Guided search: An alternative to the feature integration model for visual search. Journal of Experimental Psychology: Human Perception and Performance, 15, 419-433.

Woodman, G. F., \& Luck, S. J. (1999). Electrophysiological measurement of rapid shifts of attention during visual search. Nature, 400, 867-869.

Yantis, S. (1992). Multielement visual tracking: Attention and perceptual organization. Cognitive Psychology, 24, 295-340.

Zhang, W., \& Luck, S. J. (2009). Feature-based attention modulates feedforward visual processing. Nature Neuroscience, 12, 24-25. 